\title{
Zwischen Instanz und Dudenhausen. Der Duden heute - Einblicke in die moderne Verlagslexikografie
}

\begin{abstract}
Mit diesem Artikel möchte ich einen Überblick über die gegenwärtige Situation und die Herausforderungen geben, vor denen der traditionsreiche Dudenverlag steht, der als Imprint des Verlags Bibliographisches Institut GmbH seit 137 Jahren Wörterbücher, Grammatiken und andere Titel zum Thema deutsche Sprache publiziert. Der Umzug des Verlags von Mannheim nach Berlin im Jahr 2013 war ein tiefer Einschnitt in der Verlagsgeschichte, nach dem es galt und gilt, den Verlag wirtschaftlich wieder zu stabilisieren und Perspektiven für die Zukunft aufzuzeigen.
\end{abstract}

\section{Die vergangenen zehn Jahre}

Im Jahr 2008 verkauften die Mehrheitsaktionäre des Verlags Bibliographisches Institut \& F.A. Brockhaus AG, die Familie Langenscheidt, den Markennamen Brockhaus und die entsprechenden Inhalte an die Firma Wissenmedia in Gütersloh, ein Unternehmen der Bertelsmann-Gruppe. Damit war das Ende dieser traditionsreichen Firma mit den beiden Marken im Namen besiegelt, es verblieb am Standort Mannheim die Firma Bibliographisches Institut AG. Statt 400 Mitarbeitern kamen nun täglich nur noch 200 Menschen zur Arbeit in den Verlag, der seinen Sitz in der Dudenstraße hatte. Dieser Teilverlag wurde dann ein Jahr später von den Eigentümern ebenfalls verkauft, und zwar an den Verlag Cornelsen in Berlin. Die Cornelsen-Geschäftsführung beschloss im Jahr 2012, das Bibliographische Institut nach Berlin zu holen, also an den Stammsitz des Schulbuchverlags. Einige Verlagsteile wurden verkauft, wie z.B. der Bereich Kinder- und Jugendbuch an den S.-Fischer-Verlag in Frankfurt am Main. Es zogen also im Sommer 2013 neun Kolleginnen und Kollegen von Mannheim nach Berlin, 31 waren in Berlin neu eingestellt worden, sodass der Neustart dort mit rund 40 Leuten begann. Der Bereich Sprachtechnologie, der zunächst noch in Mannheim blieb, wurde 2014 geschlossen und damit waren rund 60 Jahre Bibliographisches Institut in Mannheim Geschichte geworden. 
Auch die ersten Berliner Jahre brachten noch einmal Veränderungen mit sich: Durch Strukturveränderungen sank die Zahl auf etwa 30 Mitarbeiterinnen und Mitarbeiter und auch der Verlagssitz wurde noch einmal verlegt: Von Treptow im Osten der Stadt ging es nach Charlottenburg in den Westen.

Die Redaktion des Verlags, die heute 11 Mitarbeiter und Mitarbeiterinnen hat, verantwortet drei große Verlagsteile: den Bereich Wörterbücher und Grammatiken, den Bereich Sachbücher und Atlanten und den Bereich Lernhilfen (Nachmittagsmarkt). Dieses Team betreut rund 1.000 Titel, von denen ein großer Teil sogenannte Backlisttitel sind. Sie erscheinen unter den Markennamen Duden (Wörterbücher, Grammatiken, Sprachratgeber, Sprachsachbücher, Namensbücher, Lernhilfen, Bewerbungsratgeber), Meyers (Sachbücher, Atlanten), Cornelsen Scriptor (Ratgeber) und Artemis und Winkler (Belletristik). Markenlizenzen wurden vergeben u.a. an die Firmen S. Fischer Verlag in Frankfurt am Main (Kinder- und Jugendbuch), Duden-Lerninstitute (Nachhilfeeinrichtung), epc (Korrektursoftware).

\section{Wie wird die Marke Duden heute wahrgenommen?}

Mithilfe von Nutzerstudien, Marktbeobachtungen und in Strategieprozessen haben wir in den ersten Monaten des Jahres 2017 ermittelt, wie Duden als Marke in Zeiten des digitalen Nachschlagens wahrgenommen wird. Das Ergebnis überraschte uns nicht: Duden wird nach wie vor mit traditionellen Werten wie

Instanz, Regeln (richtig/falsch), Sicherheit, Disziplin, Verlässlichkeit, Vertrauen, Kompetenz, Distanz, Korrektheit, Tradition, Seriosität, Sachlichkeit, Eindeutigkeit, sehr hohe Bekanntheit (im In- und Ausland)

assoziiert. Und wie ein junger Mann in den Interviews sagte: Der Duden wirkt für viele, vor allem junge Menschen, „wie aus der Zeit gefallen“.

Dieser Befund lässt nur eine Konsequenz zu: Duden muss in der Jetztzeit ankommen, wenn er auch in Zukunft eine gewichtige Rolle bei der Vermittlung von Sprachwissen spielen will. Duden muss sich also der Herausforderung stellen, moderner, aber nicht modernistisch und anbiedernd zu sein und mit seinen Produkten auch Spaß und Freude am Umgang mit der Sprache zu vermitteln. Das bedeutet, dass Duden weiterhin für die oben genannten Basiswerte steht. Aber zukünftig soll Duden auch für weitere Werte stehen:

Leidenschaft, Sprachliebe, Spaß, Kreativität, Inspiration, Partner auf Augenhöhe, Unterstützung, Leichtigkeit, Überraschung, Ideen, lustvolles Lernen. 


\section{Woran arbeiten die Redaktion und die anderen Mitarbeiterinnen und Mitarbeiter des Verlags derzeit?}

Viele Jahre wurde von der Geschäftsführung des Bibliographischen Instituts eine Programmpolitik betrieben, die keinerlei Ausbau des Programms vorsah oder diesen lediglich durch Zweit- und Drittverwertungen realisieren wollte.

Seit einem guten Jahr vollzieht der Verlag eine Wende in dieser Politik: Es erfolgt ein offensiver Ausbau der redaktionellen Bereiche, es wird in die Schaffung neuer Substanzen investiert und wir versuchen, den Übergang hin zu einem Autorenverlag zu vollziehen.

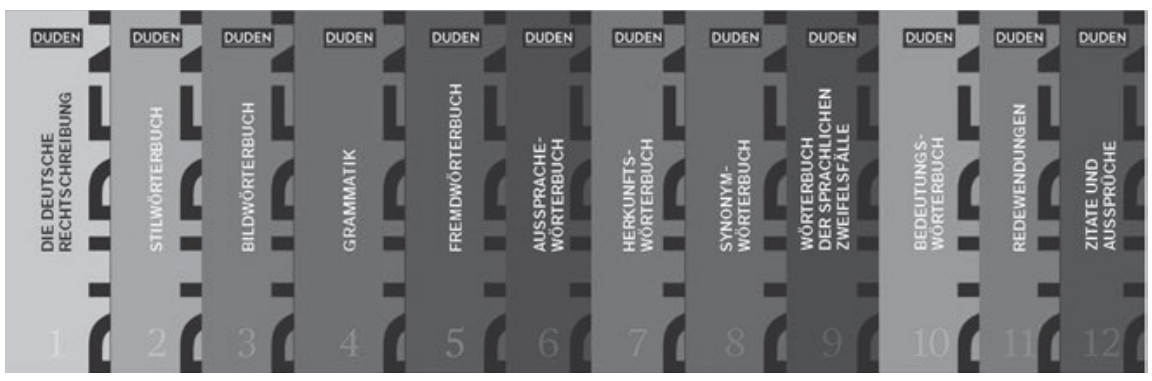

Abb. 1: Duden 1-12 in neuem Design

Eine solche Wende bringt natürlich auch Veränderungen im Verlag mit sich: Seit Jahren des Abbaus erfolgt nun wieder ein vorsichtiger und langsamer Personalaufbau. Viele strategische Unternehmungen zielen beispielsweise auf eine engere Verzahnung mit Cornelsen und auf die Erschließung neuer Geschäftsfelder. Wir arbeiten aber auch intensiv am Marken-Management-Code des Hauses, der das eigene Haus, aber auch Lizenznehmer wie S. Fischer, die Duden-Lerninstitute und epc, die Firma, die den Duden-Korrektor herstellt und vertreibt, betrifft. In diesem Zusammenhang wurde beispielsweise auch das Dudenlogo modernisiert und eine neue Gestaltung für die Reihe Duden 1-12 entworfen und umgesetzt. Ferner wurde eine neue Verlagssoftware, in der der Lebenszyklus eines Produkts von der Idee bis zur Verramschung der Restbestände (bei Printtiteln) abgebildet werden kann, eingeführt. Und im sogenannten XML-Projekt geht es um die Überführung aller Werkdaten in XML-Strukturen, die effizientere Produktionsabläufe und die Ausspielung in verschiedene Produktvarianten ermöglichen. Wir haben einen Autorenbeirat geschaffen, Gleiches ist auch noch für Händler geplant. Der 
Lizenzhandel und der Contenthandel, also der Handel mit Sprachdaten, werden intensiviert. Duden.de wird konsequent weiterentwickelt und auch für die intensivere Kommunikation mit den Kunden und anderen Sprachinteressierten genutzt. Das gilt auch für die sozialen Netzwerke.

Für die Wörterbuchredaktion bedeutet Programmausbau aber natürlich auch, dass die Zusammenarbeit mit externen Kollegen und Kolleginnen unverzichtbar ist und einen immer breiteren Raum einnehmen wird. Genauer betrachtet besteht also die eine Herausforderung darin, Lexikografinnen und Lexikografen, die unsere komplexen IT-Systeme beherrschen lernen, zu gewinnen. Die andere Herausforderung besteht in der Justierung der Qualitätssicherung.

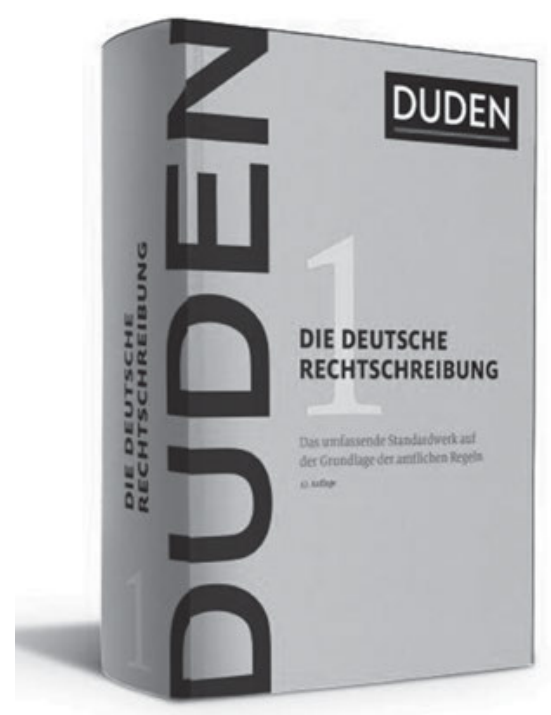

Abb. 2: Duden 1 „Die deutsche Rechtschreibung“

Beispiele für eine solche enge Zusammenarbeit mit externen Mitarbeiterinnen und Mitarbeitern sind die jeweils aktuellen Auflagen von Dudenband 4, „Die Grammatik“, Dudenband 9, „Das Wörterbuch der sprachlichen Zweifelsfälle“, und Dudenband 6, „Das Aussprachewörterbuch“. So wurden die beiden wichtigsten Schwerpunkte der redaktionellen Bearbeitung der 8. und damit aktuellen Auflage des „Wörterbuchs der sprachlichen Zweifelsfälle“ in Abstimmung zwischen Redaktion und externen Bearbeitern wie folgt festgelegt:

- Orientierung an der geschriebenen Standardsprache als Leitvarietät ausbauen,

- stärkere Korpusbasierung der Aussagen: Absicherung der (Duden-)Empfehlungen vornehmen. 
Somit entspricht der Band nun einerseits korpuslinguistisch abgesicherten Standards, andererseits büßt er die Empfehlungsfunktion nicht ein. Die sogenannten Überblicksartikel - einspaltig gesetzte umfangreichere Artikel - sind ohne Empfehlungen für eine bestimmte Variante eines bestimmten sprachlichen Phänomens abgefasst, die Einzelartikel enthalten bei Vorliegen eines Zweifelsfalls eine Empfehlung.

Bei der Bearbeitung von zwei Titeln haben wir sehr eng mit dem Institut für Deutsche Sprache zusammengearbeitet. So beim Dudenband 6, „Das Aussprachewörterbuch“, das von Stefan Kleiner und Ralf Knöbl federführend bearbeitet wurde und das nun das erste Mal mit beiden Logos, also dem des Verlags und dem des IDS, erschien, und beim Dudenband 4, „Die Grammatik“. Für diesen Band war Angelika Wöllstein zum ersten Mal als Herausgeberin tätig.

\section{Neue Werte und Programmausbau}

Ich hatte bereits die neuen Werte genannt, für die Duden in Zukunft stehen möchte: Leidenschaft, Sprachliebe, Spaß, Kreativität, Inspiration, Partner auf Augenhöhe, Unterstützung, Leichtigkeit, Überraschung, Ideen, lustvolles Lernen. Aber auf welchem Weg, mit welchen Produkten und Aktivitäten können wir diese Werte zum Leben erwecken?

Wir haben in den vergangenen Monaten Bücher aufgelegt, die heißen: „Versunkene Wortschätze. Eine Kiste voller Raritäten“, „Wolke sieben. Sprache erzählt die schönsten Geschichten“, „Du hast das Wort, Schätzchen. 100 charmante Geschichten rund um die Sprache“, „Die Tomate und das Paradies“ und „Mit Feuereifer und Herzenslust. Wie Luther unsere Sprache prägte“. Schon an den Titeln erkennt man, dass es hier nicht um Wörterbücher und Grammatiken geht, sondern um Titel, die Sprachwissen auch mal auf leichte, amüsante oder augenzwinkernde Art vermitteln. Solche Titel verlangen, um im Buchhandel bestehen zu können, natürlich eine komplett andere Art der Gestaltung und Platzierung. Hier sind also auch die Abteilungen Herstellung, Marketing und Presse noch einmal ganz anders gefordert als sonst.

\section{Weitere Herausforderungen}

Die Dudenredaktion steht vor zahlreichen weiteren Herausforderungen, die Zeit, Kraft und auch Geld kosten. So arbeiten Vertreter der Redaktion beispielsweise mit im 
- Rat für deutsche Rechtschreibung,

- Deutschen Institut für Normung (DIN) und

- Ständigen Ausschuss für geografische Namen (StAGN).

Es gilt aus unserer Sicht aber auch, Duden neu zu positionieren im Umfeld von Sprachpflege und -kultur. Auch dazu werden neue Titel entwickelt, u.a. die Duden-Streitschriften, die erstmals 2018 erscheinen werden.

\section{Duden online}

Seit fünf Jahren ist Duden die bekannteste Marke auch im Bereich (deutsche) Onlinelexikografie. Das kostenlose Angebot ist seit 2012 im Netz und seitdem schreibt diese Website Erfolgsgeschichte. Diese Aussage können wir belegen mit den folgenden Zahlen:

- Im Februar 2017 besuchten 12 Millionen Nutzer die Seite, sie erzeugten 45 Millionen Seitenzugriffe.

- Pro Jahr sind 130 Millionen Besucher mit rund einer halben Milliarde Zugriffe auf unserer Seite unterwegs.

- In Spitzenzeiten sind 6.500 Nutzer gleichzeitig auf der Seite.

- Die Zugriffe erfolgen aus nahezu allen Ländern der Welt, nach Deutschland, Österreich und der Schweiz sind am stärksten die USA und Großbritannien vertreten.

- $\quad$ Rund 70\% der Nutzer und Nutzerinnen sind zwischen 18 und 34 Jahre alt.

- Fast 60\% sind weiblich.

- $60 \%$ der Nutzer und Nutzerinnen kommen wieder, sie nutzen die Plattform bis zu zehnmal am Tag.

Auch, aber nicht nur über diese Seite kommunizieren die Kundinnen und Kunden mit uns:

Uns erreichen ca. 30 Kundenzuschriften am Tag (und das sind nur inhaltliche Rückmeldungen zu Duden online oder unseren Titeln). Deren Beantwortung erfordert rund 1200 Stunden Arbeitszeit, was etwa einer Vollzeitstelle entspricht. In der DudenSprachberatung gehen täglich ca. 70 Anrufe ein. Der Newsletter der Duden-Sprachberatung erscheint monatlich, rund 40.000 Menschen haben ihn abonniert. 


\section{Duden auf Facebook}

Duden ist seit Januar 2012 auf Facebook aktiv. Die Seite hat inzwischen über 135.000 Follower und damit die größte Reichweite im Verlagsbereich überhaupt. Mit unseren Posts erreichen wir bis zu 1,7 Millionen Menschen, die durchschnittliche Reichweite liegt bei 30.000. Die Seite wächst schnell, aber organisch: Pro Woche gewinnen wir rund 700 neue Follower. Am wichtigsten ist aber vielleicht, dass unsere Follower sehr aktiv sind, das heißt, 15 bis $20 \%$ von ihnen liken, teilen und kommentieren unsere Beiträge.

Einer der Höhepunkte der Öffentlichkeitsarbeit in den letzten Monaten bestand für uns darin, am Heft „Micky Maus genial - Sprache“ mitarbeiten zu können. Dieser Ausflug nach Dudenhausen war eine bereichernde Erfahrung für die beteiligten Redakteurinnen und Redakteure, ist es doch alles andere als einfach, einen Text nach Micky-Maus-Maßstäben zu verfassen und mit ihm Wissen über Sprache zu transportieren.

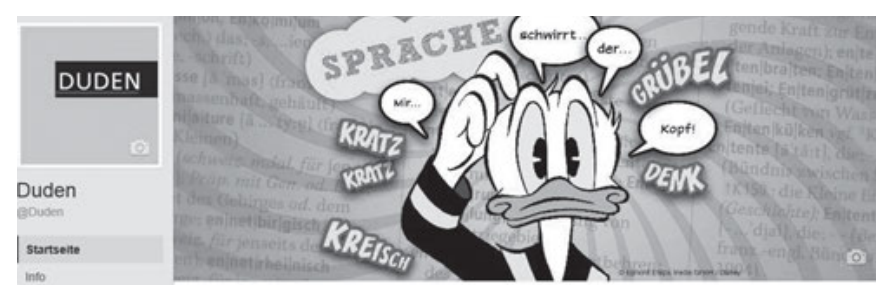

Abb. 3: Zusammenarbeit zwischen Duden und „Micky Maus“

\section{Zusammenfassung}

Ich habe versucht $\mathrm{zu}$ zeigen, vor welchen vielfältigen Herausforderungen die Dudenredaktion und der Dudenverlag in diesen Jahren stehen. Diese Herausforderungen betreffen sowohl Strukturen, Inhalte, Positionierungen, Gestaltung, Kundenservice und vieles andere. Besonders wichtig sind uns dabei

- die Sicherung des Stellenwerts der Marke Duden,

- der Ausbau des Programms im Printbereich,

- die Gestaltung des digitalen Wandels (Entwicklung und Vertrieb von Produkten wie Apps, Software und Sprachdienstleistungen) sowie

- der Ausbau des Kundendienstes.

Unser Ziel ist es, dafür zu sorgen, dass Duden auch in Zukunft DER Ansprechpartner zum Thema (deutsche) Sprache ist. 